\title{
Application of Spatial Domain Interferometry with the Capon Method to Transcranial Doppler Ultrasonography: a Simulation Study
}

\author{
Shigeaki Oкumura, , ${ }^{*}$ Aya Kita, ${ }^{* *}$ Hirofumi Taki, ${ }^{*}$ Yoshiki Nagatani, ${ }^{* * *}$ Mami MatsuKawa, ${ }^{\dagger}$ Toru Sato
}

\begin{abstract}
Control of vasospasm is one of the most important problems in postoperative management after the occurrence of subarachnoid hemorrhage. Transcranial Doppler ultrasonography (TCD) is a non-invasive test that measures cerebral blood flow. However, high-intensity interference returned from the cranium causes estimation errors. A moving target indicator (MTI) filter is widely used to suppress the interference. The MTI filter suppresses only static target echoes, and hence the time-varying interference component caused by movement of the probe remains. To suppress the time-varying component, we apply spatial domain interferometry (SDI) with the Capon method to the MTI filtered signal. The method suppresses interference by minimizing the output power under the constraint condition of a constant response from a desired direction. The method requires estimation of the covariance matrix between signals received at the elements by averaging independent data. Conventional imagers based on SDI with the Capon method average the matrix in the temporal direction only in order to achieve high axial resolution. In TCD, both high temporal resolution and sufficient accuracy in measuring blood flow velocity are desired. Therefore, we propose a technique that averages the covariance matrix in both temporal and axial directions. We evaluated the performance of an SDI imager using the proposed technique in a simulation study, in which the array size was 12 elements, the transmit center frequency was $2.0 \mathrm{MHz}$, and the temporal and axial averaging lengths were $0.70 \mathrm{~ms}$ and $5.6 \mathrm{~mm}$, respectively. The ratio of desired signal intensity to cranium interference intensity was $-40 \mathrm{~dB}$. The delay and sum (DAS) beamformer failed to estimate blood flow velocity of $1.0 \mathrm{~m} / \mathrm{s}$, and estimation error and standard deviation of 1.9 and $0.92 \mathrm{~m} / \mathrm{s}$, respectively. When the size for spatial averaging ranged from $25 \%$ to $50 \%$ of the number of elements, the proposed SDI beamformer succeeded to estimate the velocity of $1.0 \mathrm{~m} / \mathrm{s}$ with estimation error and standard deviation of $0.044 \mathrm{~m} / \mathrm{s}$ and $0.035 \mathrm{~m} / \mathrm{s}$, respectively. In contrast, the conventional SDI beamformer had estimation error and standard deviation of 0.17 and $0.25 \mathrm{~m} / \mathrm{s}$, respectively. These results indicate the effectiveness of the proposed technique in applying the SDI imaging method to TCD.
\end{abstract}

Keywords: transcranial Doppler ultrasonography, spatial domain interferometry, adaptive beamforming, blood flow velocity estimation, Capon method.

Adv Biomed Eng. 4: pp. 73-79, 2015.

\section{Introduction}

Subarachnoid hemorrhage ( $\mathrm{SAH}$ ), bleeding inside the cranium, is one of the most serious and important conditions faced by neurosurgeons, because between $20 \%$ and $40 \%$ of SAH patients have poor outcomes $[1,2]$. One of the main causes of poor outcome is vasospasm that reduces the blood flow in the middle cerebral artery and induces serious conditions such as brain infarct. The period with the highest risk for vasospasm is from 3 to 14 days after $\mathrm{SAH}$, and it is essential to carry out a daily test for vasospasm [2].

Several monitoring techniques have been reported for the

This study was presented at the Symposium on Biomedical Engineering 2014, Tokyo, September, 2014.

Received on August 1, 2014; revised on November 1, 2014; accepted on January 20, 2015.

* Graduate School of Informatics, Kyoto University, Kyoto, Japan.

** Sakai Rumi Clinic, Hyogo, Japan.

*** Department of Electronics, Kobe City College of Technology, Hyogo, Japan.

${ }^{\dagger}$ Faculty of Science and Engineering, Doshisha University, Kyoto, Japan.

\# Yoshida-Hommachi, Sakyoku, Kyoto 606-8501, Japan.

E-mail: sokumura@sato-lab.0t0.jp detection of vasospasm. Three-dimensional computed tomography (3D-CT) and magnetic resonance angiography provide high-resolution images in the cranium $[3,4]$. However, it is difficult to perform these tests repeatedly because of their invasiveness. In contrast, transcranial Doppler ultrasonography (TCD) is a non-invasive and repeatable test that measures the blood flow velocity in the middle cerebral artery $[1,5]$. While TCD is performed to detect the occurrence of vasospasm after SAH, its low reliability prevents it from playing a primary role [1].

One of the reasons for the low reliability of TCD is the low signal-to-noise ratio, caused by high-intensity interference returned from the cranium. To suppress the effect of the interference, ultrasound imagers usually employ a clutter filter named a moving target indicator (MTI) filter [6-8]. However, the MTI filter only suppresses the echoes from stationary targets [9]. Therefore, the movement of an ultrasound probe is one of the causes of error in estimating blood flow using TCD. Several researchers have reported devices that fix an ultrasound probe to the cranium $[10,11]$. However, these devices are unsuitable for postoperative management after brain surgery.

To improve the performance of TCD in blood flow velocity measurement, after MTI filtering, the time-varying interference components should be suppressed. The Capon method is an adaptive signal processing method for suppressing high-intensity interferences. The strategy of this method is to minimize the output 
power under the constraint that maintains the response of a desired signal [12-14]. The Capon method does not work when sources are strongly correlated. In ultrasonography, imagers based on the Capon method employ spatial averaging to suppress the correlation [15-18]. In TCD, the echo from red blood cells is supposed to have low correlation with interferences from the cranium, because the echo from blood cells is a Doppler signal returned from randomly distributed scatterers, whereas interference from the cranium is a non-Doppler signal returned from a hard target. Therefore, the size for spatial averaging in TCD may be smaller than that in typical ultrasonography.

The Capon method requires estimation of the covariance matrix that expresses the cross-correlation between two signals at two elements. A conventional spatial domain interferometry (SDI) beamformer with the Capon method averages the covariance matrix in the temporal direction only to achieve high axial resolution. In TCD, both high temporal resolution and sufficient accuracy in measuring blood flow velocity are desired. Therefore, we propose a technique that averages the covariance matrix in both temporal and axial directions. We examined the performance of an SDI beamformer using the proposed technique applied to $\mathrm{TCD}$, and investigated the appropriate size for spatial averaging in TCD.

\section{Materials and Methods}

\subsection{Moving target indicator filter}

We first use an MTI filter to suppress the interference from the cranium. In the simulation study, we consider high-intensity multiple reflections inside the cranium because they are thought to be the major cause of the low robustness of TCD. In this case, the received signal is expressed by

$$
\begin{aligned}
& s_{k, n}(t)=b_{k, n}(t)+i_{k, n}(t), \\
& i_{k, n}(t)=i_{\mathrm{S} k}(t)+i_{\mathrm{T} k, n}(t),
\end{aligned}
$$

where $s_{k, n}(t)$ is the received signal at the $k$-th element for the $n$-th transmit event, $b_{k, n}(t)$ is the desired signal from the red blood cells, $i_{k, n}(t)$ is the interference from the cranium, $i_{\mathrm{S} k}(t)$ is the static component of the interference from the cranium, and $i_{\mathrm{T} k, n}(t)$ is the time-varying component of the interference. The movement of an ultrasound probe is the source of the time-varying component of echoes. Therefore, in blood flow measurement using TCD without a fixation device, high-intensity echo from the cranium should have a time-varying component.

The MTI filter subtracts the previously received signal from the latest received signal.

$$
\begin{aligned}
y_{\mathrm{M} k, n}(t) & =s_{k, n+1}(t)-s_{k, n}(t) \\
& =b_{k, n+1}(t)-b_{k, n}(t)+i_{\mathrm{T} k, n+1}(t)-i_{\mathrm{T} k, n}(t),
\end{aligned}
$$

where $y_{\mathrm{M} k, n}(t)$ is the output of the MTI filter. As shown in Eq. (3), the time-varying interference component still exists in the MTI-filtered signal.

\subsection{Spatial domain interferometry with the Capon method for Doppler Imaging}

To suppress the time-varying interference component, we apply SDI with the Capon method to the signals after MTI filtering. The Capon method is an adaptive beamforming technique used in high-resolution imaging [12-18]. SDI is a technique for estimating the echo intensity from a desired angle, as shown in Fig. 1. Combining SDI with the Capon method minimizes the contribution of interference from undesired directions by restricting to a

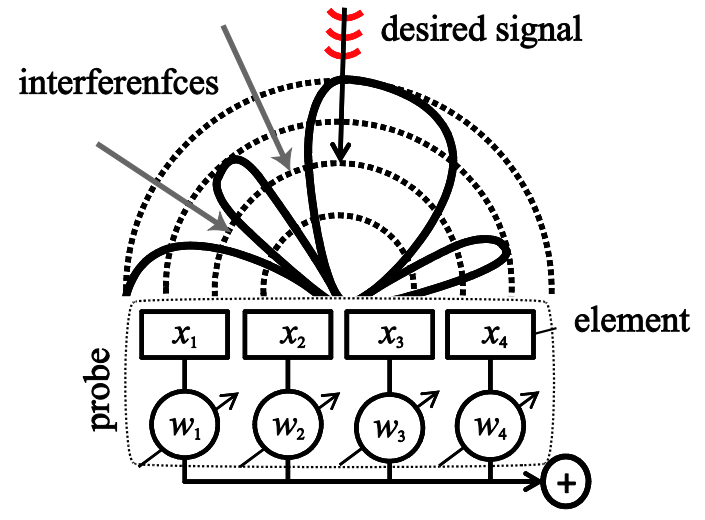

Fig. 1 Schematic presentation of SDI.

constant response from a desired direction.

When we employ a linear array composed of $K$ elements, the output of the SDI method $y_{\mathrm{SDI} n}(t)$ is given by:

$$
\begin{aligned}
& y_{\mathrm{SDI} n}(t)=\mathbf{W}^{\mathrm{H}} \mathbf{y}_{\mathrm{M} n}(t), \\
& \mathbf{y}_{\mathrm{M} n}(t)=\left[\begin{array}{lll}
y_{\mathrm{M} 1, n}^{\prime}\left(t-\Delta_{1}\right) & \cdots & y_{\mathrm{M} K, n}^{\prime}\left(t-\Delta_{K}\right)
\end{array}\right]^{\mathrm{T}},
\end{aligned}
$$

where $\mathbf{W}$ is a weighting vector, $y_{\mathrm{M} k, n}^{\prime}(t)$ is the received signal with the delay time for focusing $\Delta_{k},{ }^{\mathrm{H}}$ denotes the Hermitian operator, and ${ }^{\mathrm{T}}$ denotes transpose. This time-delay approach is the same as the conventional delay-and-sum (DAS) approach [16, 18]. In this study, we set the desired direction as the front direction perpendicular to the probe, as shown in Fig. 1. Use of the time-delay process given by Eq. (5) allows measurement of blood flow velocity at an arbitrary location. When a narrow-band signal is used, Eq. (5) can be rewritten using a steering vector. However, medical ultrasound imagers typically transmit wide-band signals. We use the time-delay approach to eliminate the error caused by the bandwidth.

The Capon method is an adaptive signal processing method that suppresses the contribution from interference by calculating the optimal weighting vector. After the time-delay process, the SDI beamformer with the Capon method is expressed by

$\min _{\mathbf{W}} \mathbf{W}^{\mathrm{H}} \mathbf{R W} \quad$ subject to $\mathbf{1} \mathbf{W}=1$,

where $\mathbf{R}$ is a covariance matrix and $\mathbf{1}=\left[\begin{array}{llll}1 & 1 & \cdots & 1\end{array}\right]$ is a constraint vector. This problem can be solved using the Lagrange multiplier methodology [16, 18]:

$$
\mathbf{W}_{\mathrm{opt}}=\frac{1}{\mathbf{1}^{\mathrm{T}}(\mathbf{R}+\eta \mathbf{E})^{-1} \mathbf{1}}(\mathbf{R}+\eta \mathbf{E})^{-1} \mathbf{1},
$$

where $\mathbf{W}_{\text {opt }}$ is an optimal weighting vector and $\eta \mathbf{E}$ is a diagonal loading matrix to obtain the inverse matrix $\mathbf{R}$ stably [13-18].

In the conventional SDI method, the covariance matrix is normally calculated by temporal-averaging $[16,18]$, because axial averaging reduced axial resolution. TCD requires high temporal resolution and high accuracy in measuring blood flow velocity. In this study, we propose a technique that averages the covariance matrix in both temporal and axial directions. As shown in Fig. 2, the axial- and temporal-averaging lengths are the signal length and the number of differential signals used for estimation, respectively. This strategy introduces the assumption that interference arrival directions and blood flow velocity in the desired direction are constant in temporal and axial directions. This assumption allows averaging of the covariance matrices across successive dif- 
ferential signals. When we use $N$ signals for estimation, we acquire $N-1$ differential signals. Accordingly, the $(l, m)$-th element of the expected covariance matrix $\mathbf{R}$ with spatial averaging is defined by

$r_{l, m}=\frac{1}{(N-1) t_{\mathrm{a}}} \sum_{k_{\mathrm{s}}=1}^{K_{\text {ave }}} \sum_{n=1}^{N-1} \int_{t_{\mathrm{d}}-t_{\mathrm{a}} / 2}^{t_{\mathrm{d}}+t_{\mathrm{a}} / 2} y_{\mathrm{M} k_{\mathrm{s}}+l-1, n}(t) y_{\mathrm{M} k_{\mathrm{s}}+m-1, n}^{*}(t) \mathrm{d} t$,

where $N$ is the number of signals used for the method, $t_{\mathrm{a}}$ is the axial-averaging length, $t_{\mathrm{d}}$ is the time of measurement depth, ${ }^{*}$ denotes the complex conjugate, and $K_{\text {ave }}$ is the size for spatial averaging. In this setting, axial and temporal resolutions become $c t_{\mathrm{a}} / 2$ and $N T$, respectively. Here $c$ is the speed of sound and $T$ is the pulse repetition time.

We used an axial-averaging length $t_{\mathrm{a}}$ of $7.5 \mu \mathrm{s}$ in the simulation. This parameter assumes that the middle cerebral artery runs at least $5.6 \mathrm{~mm}$ along the measurement direction. According to clinical studies [19-21], the middle cerebral artery ranges in length from 14 to $16 \mathrm{~mm}$. Therefore, our averaging length is reasonable under clinical conditions.

In this study we set $N=8$, i.e., the temporal resolution was $8 T$. When the maximum measurement depth is $6.5 \mathrm{~cm}$, the pulse repetition time $T$ is $87 \mu$ s. This setting assumes that blood flow velocity and the direction of interference are constant for $0.70 \mathrm{~ms}$, and allows the acquisition of a 2-D image consisting of 60 scan lines with an acquisition rate of 24 frames/s. This temporal resolution is acceptable for monitoring purpose.

\subsection{Velocity estimation method}

Blood flow velocity is estimated by calculating the phase rotation between two successive signals received at the center frequency [22]. When the red blood cells move forward to the probe at a constant velocity of $v_{\mathrm{d}}(t)$, the phase rotation $\phi(t)$ is given by:

$$
\phi(t)=\frac{4 \pi v_{\mathrm{d}}(t) T}{\lambda},
$$

where $\lambda$ is the wavelength at the center frequency. Therefore, the estimated blood flow velocity $v_{\mathrm{e}}$ is expressed by

$$
\begin{aligned}
& v_{\mathrm{e}}=\frac{\lambda \overline{\phi_{\mathrm{e}}}}{4 \pi T}, \\
& \overline{\phi_{\mathrm{e}}}=\arg \sum_{n=1}^{N-2} \int_{t_{\mathrm{d}}-t_{\mathrm{a}} / 2}^{t_{\mathrm{d}}+t_{\mathrm{a}} / 2} y_{\mathrm{SDI} n+1}(t) y_{\mathrm{SDI} n}{ }^{*}(t) \mathrm{d} t
\end{aligned}
$$

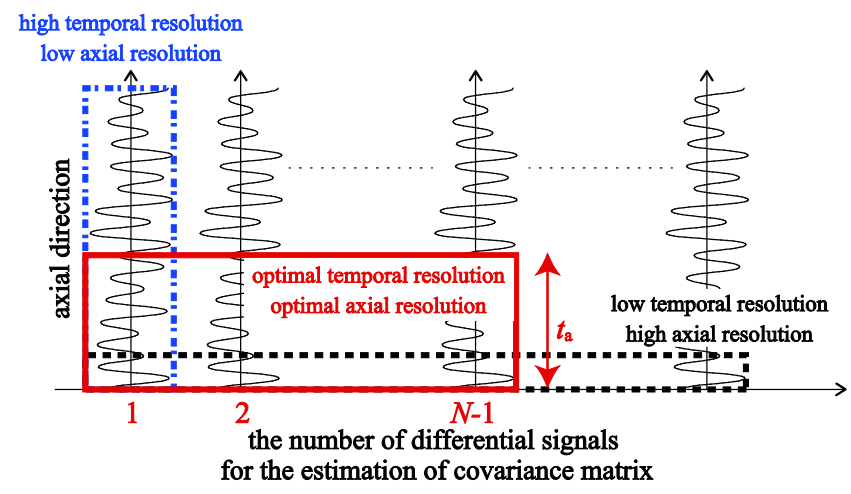

Fig. 2 Schematic presentation of temporal- and axial-averaging in estimating the expected covariance matrix.

\section{Results}

\subsection{Estimation of intensity and correlation of interference from the cranium}

To evaluate the proposed method, estimation of the ratio of signal intensity to intensity of the interference returned from the cranium $\left(\mathrm{SI}_{\mathrm{C}} \mathrm{R}\right)$ is required. We thus estimated the $\mathrm{SI}_{C} \mathrm{R}$ in a realistic model using an elastic 3D finite-difference time-domain (FDTD) simulation [23-25] and in an experiment. The schematic presentation of an FDTD simulation is shown in Fig. 3(a). A wave propagation pattern in FDTD is shown in Fig. 3(b). The parameters of material properties employed in FDTD simulation are shown in Table 1. The total simulation field was $7.5 \times 37 \times 1.8 \mathrm{~mm}$. The size of the cube lattice $\Delta x$ was $46 \mu \mathrm{m}$. A point source transmitted an ultrasound pulse; the center frequency of the pulse was set at 2.0 MHz according to a previous study [26], and the pulse length was set at $5.0 \mu \mathrm{s}$. The distance between the transmit point and the cranium surface was $5.0 \mathrm{~mm}$, and the thicknesses of the cancellous bone layer and the cortical bones were $3.0 \mathrm{~mm}$ and $1.0 \mathrm{~mm}$, respectively. The 3D data of the cancellous bone layer was constructed using the 3D data of a bovine sample acquired using an X-ray CT imager [27-29]. In the FDTD simulation, we ignored the effect of absorption of the medium.

We first estimated the amplitude of the interference returned from the cranium based on the received signal at $\mathrm{R}_{0}$. We fitted an exponential curve tangential to the envelope of the received signal in the range from 15 to $30 \mu$ s, as shown in Fig. 4. If we assume that the amplitude of the interference decreases exponentially, the amplitude of the interference may be smaller than that of the fitted curve. In the FDTD simulation, the amplitude of the fitted curve at a desired depth of $5.0 \mathrm{~cm}$ was $3.0 \times 10^{-7}$ times the maximum amplitude at the transmit point $\mathrm{R}_{0}$.

We next estimated the amplitude of blood echo in the cranium using an experimental study and an FDTD simulation. In the experimental study, we investigated the ratio of amplitude of

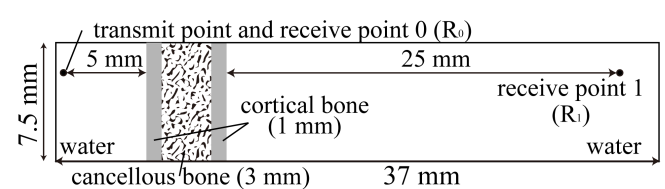

(a)

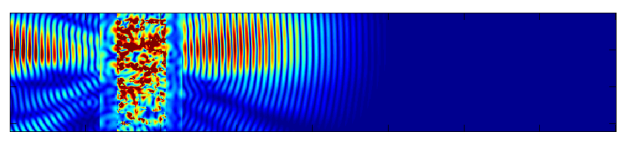

(b)

Fig. 3 (a) Schematic of an FDTD simulation and (b) a wave propagation pattern.

Table 1 Parameters of FDTD simulation.

\begin{tabular}{lll}
\hline \hline Material & Water & Bone \\
\hline Density $\left[10^{3} \mathrm{~kg} / \mathrm{m}^{3}\right]$ & 1.0 & 2.0 \\
Velocity $[\mathrm{km} / \mathrm{s}]$ & 1.5 & 4.4 \\
Lame's coefficients $\lambda[\mathrm{GPa}]$ & 2.3 & 20 \\
Lame's coefficients $\mu[\mathrm{GPa}]$ & 0.0 & 9.4 \\
\hline \hline
\end{tabular}




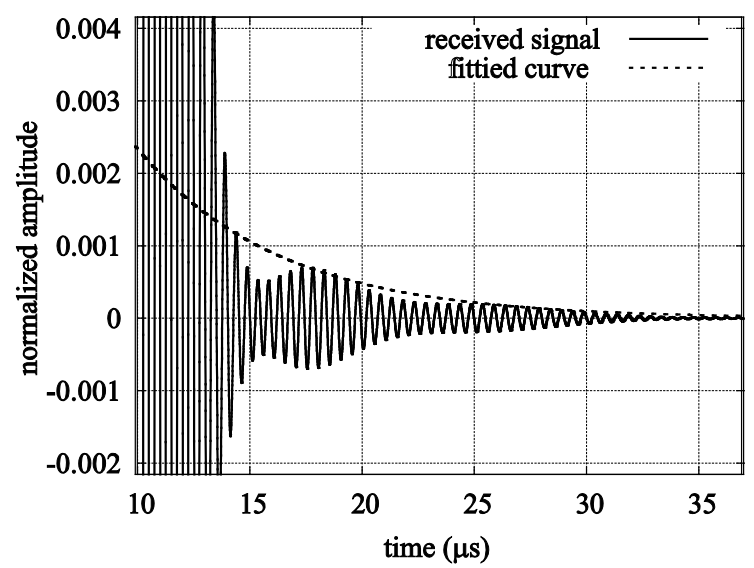

Fig. 4 Received signal at receive point $\mathrm{R}_{0}$ and the fitted curve in the FDTD simulation.

blood echo to muscle layer echo. In the FDTD simulation, we estimated the amplitude of the muscle layer in the cranium. In the experiment, we compared the amplitude of the echo from swine blood with that from swine muscle layer. From two blood signal data sets and four muscle tissue data sets, the average ratio of blood echo intensity to muscle echo intensity was $29 \mathrm{~dB}$ with standard deviation of $2.5 \mathrm{~dB}$. In the FDTD simulation, we expressed the echo amplitude of muscle layer in the cranium $A_{\mathrm{TC}}(t)$ as follows:

$$
A_{\mathrm{TC}}(t)=\gamma A^{2}(t) \sqrt{\frac{S}{\Delta x^{2}}},
$$

where $\gamma=0.053$ is the reflectance between connective tissue and muscle, $S$ is the square of the beam spot, and $A(t)$ is the amplitude of the received signal at the receive point $\mathrm{R}_{1}$. In Eq. (12), we assume that the signal is integrated incoherently within the beam width. We used a linear array probe consisting of 12 elements. Each element pitch was $1.0 \mathrm{~mm}$, i.e., the whole probe aperture was $12 \mathrm{~mm}$, which was the same setting as used in a previous study [30]. Because the center frequency was $2.0 \mathrm{MHz}$ and the aperture of the probe was $12 \mathrm{~mm}$, we supposed that $S=7.7 \mathrm{~mm}^{2}$. Under this condition, the average and standard deviation of blood signal amplitude in the cranium were $3.9 \times 10^{-9}$ and $1.1 \times 10^{-9}$ times, respectively, the maximum amplitude at the transmit point $R_{0}$. As a result, the average estimated $\mathrm{SI}_{C} \mathrm{R}$ was $-34 \mathrm{~dB}$ and standard deviation was $2.6 \mathrm{~dB}$.

The velocity in surrounding tissue and the blood velocity in a peripheral vessel are slower than that in the middle cerebral artery. Because the difference in blood velocity results in difference of Doppler velocity, the echo from a peripheral vessel would have low correlation with the echo from the middle cerebral artery. In this section, we investigated the cross-correlation between the echo from the middle cerebral artery and that from the cranium in a simulation study using FDTD.

The echo from blood scatterers was calculated using the Field II simulation package [31,32]. We set randomly distributed targets at a depth of $50 \mathrm{~mm}$, with axial and lateral velocities of $1.0 \mathrm{~m} / \mathrm{s}$ and $0.50 \mathrm{~m} / \mathrm{s}$, respectively. We constructed the echo from the cranium using the FDTD data at $\mathrm{R}_{0}$ after $35 \mu$ s because the direct wave should have no effect on the data after $35 \mu \mathrm{s}$. The cross-correlation coefficient between the echo from blood scatter-

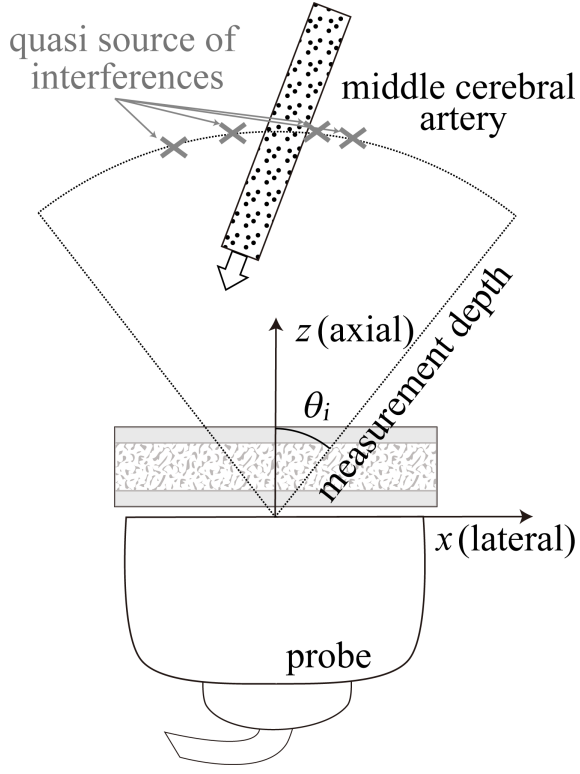

Fig. 5 Schematic presentation of the simulation model.

ers and the echo from the cranium was 0.072 in the TCD measurement setting. This result shows that in TCD, the desired signal has a sufficiently low correlation with interference, indicating that the size for spatial averaging in TCD may be smaller than that in normal tissue imaging.

\subsection{Simulation setting}

In the simulation study, we used the Field II simulation package [31-33]. A schematic presentation of the simulation is shown in Fig. 5. Because the diameter of the middle cerebral artery is about $3 \mathrm{~mm}$, moving scatterers in a vessel are randomly distributed in the region of $|x|<1.5 \mathrm{~mm}$ and $35 \mathrm{~mm}<z<65 \mathrm{~mm}$, where scatterer density is $5 / \mathrm{mm}^{2}$. The blood scatterers move forward toward the probe and have a constant velocity of $1.0 \mathrm{~m} / \mathrm{s}$ in the axial direction and $0.50 \mathrm{~m} / \mathrm{s}$ in the lateral direction.

In this study, we focused on measuring the blood flow velocity of the middle cerebral artery. The middle cerebral artery is one of the three major artery pairs that supply blood to the cerebrum. Other peripheral vessels in the temporal region are much narrower than the middle cerebral artery. Since the echo intensity of a peripheral vessel is much lower than that of the middle cerebral artery, detection of a peripheral vessel requires a contrast agent [34]. In this study, we thus neglected the effect of the echoes from peripheral vessels.

We assumed that the cranium interference can be represented by the sum of the signals from four point sources for the depth of measurement. The sources emitted signals equivalent to that obtained by FDTD at $\mathrm{R}_{0}$ after $35 \mu \mathrm{s}$. The directions of arrival of interference $\theta_{i}$ were $-15,-7.5,5.0$, and 10 degrees. We also assumed that the time-varying component of the interference from the cranium is given by:

$$
I_{\mathrm{T} n}(\omega)=I_{\mathrm{S} n}(\omega) \alpha_{\mathrm{g} n} \mathrm{e}^{\mathrm{j} \theta_{\mathrm{r}}},
$$

where $I_{\mathrm{T} n}(\omega)$ and $I_{\mathrm{S} n}(\omega)$ are the Fourier transforms of $i_{\mathrm{T} n}(t)$ and $i_{\mathrm{S} n}(t)$, respectively, and $\alpha_{\mathrm{g} n}$ is a random number generated from a Gaussian distribution with zero mean. In this study, we set the standard deviation $\alpha_{\mathrm{g} n}$ to be $1.0 \times 10^{-2}$. In other words, the intensity of the time-varying interference component is $40 \mathrm{~dB}$ lower 


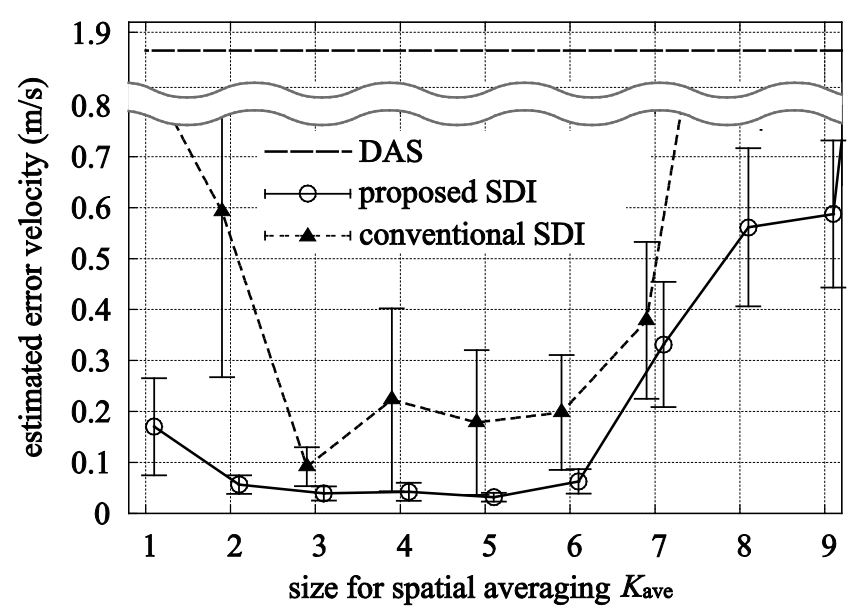

Fig. 6 Blood flow velocities estimated using a DAS with an MTI filter, and the proposed SDI beamformer, and a conventional SDI beamformer, where the size for spatial averaging ranged from 1 to 9 and $\mathrm{SI}_{\mathrm{C}} \mathrm{R}=-40 \mathrm{~dB}$. The true blood-flow velocity is $1.0 \mathrm{~m} / \mathrm{s}$. Error bars show standard deviations. The standard deviation of estimated error velocity of DAS was $0.92 \mathrm{~m} / \mathrm{s}$.

than that of total interference, and the phase $\theta_{\mathrm{r}}$ is a random number that follows a uniform distribution between 0 and $2 \pi$.

We used a linear array probe consisting of 12 elements. Each element pitch was $1.0 \mathrm{~mm}$. We set the value of the diagonal loading term $\eta$ to be $10 \mathrm{~dB}$ lower than the average intensity of the desired signal returned from red blood cells. The center frequency of the pulse was set at $2.0 \mathrm{MHz}$, and the pulse length was set at $5.0 \mu \mathrm{s}$.

\subsection{Estimation accuracy of the proposed SDI beamformer and spatial averaging technique}

Figure 6 shows the estimation error of blood flow velocity using a conventional DAS beamformer with an MTI filter, the proposed SDI beamformer using temporal- and axial-averaging, and the conventional SDI beamformer using only temporal averaging. We calculated the flow velocity in 10 samples, where $\mathrm{SI}_{\mathrm{C}} \mathrm{R}$ was $-40 \mathrm{~dB}$. The DAS beamformer focused at the center of the ROI, i.e. the focal depth was $50 \mathrm{~mm}$.

The conventional DAS beamformer failed to estimate blood flow velocity of $1.0 \mathrm{~m} / \mathrm{s}$, and estimation error and standard deviation of blood flow velocity were $1.9 \mathrm{~m} / \mathrm{s}$ and $0.92 \mathrm{~m} / \mathrm{s}$, respectively. The conventional SDI beamformer performed better than the DAS beamformer; however, the estimation accuracy was acceptable only when the size for spatial averaging was 3 . In contrast, the SDI beamformer using the proposed technique showed excellent and stable performance when the size for spatial averaging ranged from 2 to 6 . When the size for spatial averaging ranged from 3 to 6, the proposed SDI beamformer succeeded in estimating the velocity of $1.0 \mathrm{~m} / \mathrm{s}$ with estimation error and standard deviation of $0.044 \mathrm{~m} / \mathrm{s}$ and $0.035 \mathrm{~m} / \mathrm{s}$, respectively, compared with $0.17 \mathrm{~m} / \mathrm{s}$ and $0.25 \mathrm{~m} / \mathrm{s}$ for the conventional SDI beamformer. This result indicates the effectiveness of the proposed technique in applying the SDI beamformer to TCD.

\section{Discussion}

In this study, we focused on the characteristics of TCD and pro-

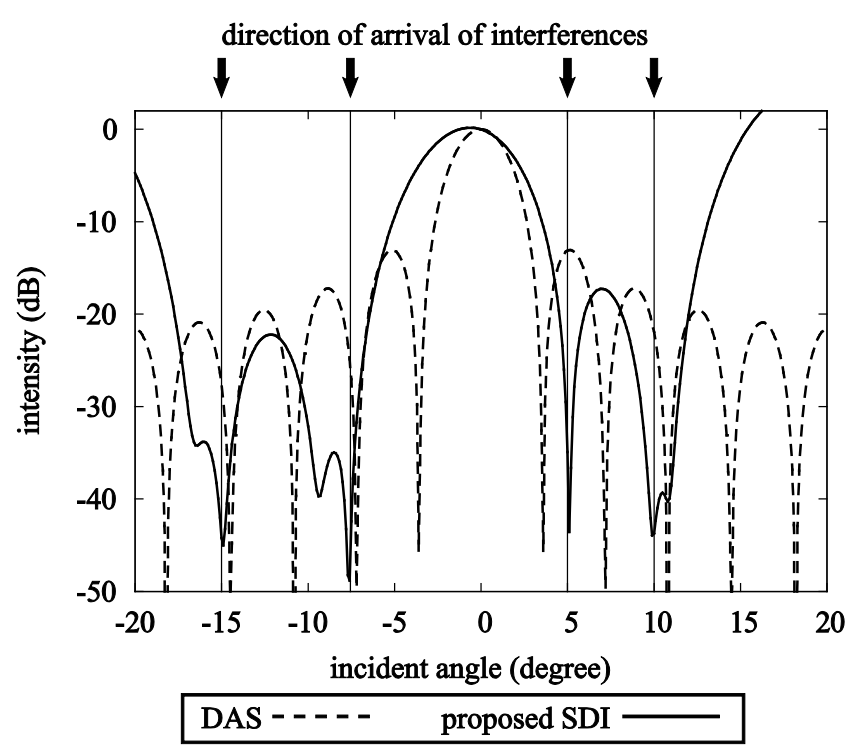

Fig. 7 Beam pattern of the conventional delay-and-sum beamformer and that of the proposed SDI beamformer with size for spatial averaging $K_{\text {ave }}$ of 5 at center frequency, where $\mathrm{SI}_{\mathrm{C}} \mathrm{R}=-40 \mathrm{~dB}$. The directions of arrival of interferences were $-15,-7.5,5.0$, and 10 degrees.

posed an adaptive signal processing method for TCD that suppresses the effect of high-intensity cranium echo. We evaluated the proposed SDI beamformer that employs temporal- and axial-averaging, and investigated the performance of spatial averaging by the proposed method in Doppler imaging in a simulation study. The result shown in Fig. 6 suggests that the appropriate size for spatial averaging may be from $25 \%$ to $50 \%$ of the number of elements $K$. In typical SDI beamformers, the size for spatial averaging is from $50 \%$ to $75 \%$ of the number of elements [16]. This result indicates that in TCD, the appropriate size for spatial averaging is smaller than that in normal tissue imaging. This finding is consistent with the prediction described in Section 1 that the size for spatial averaging in TCD may be smaller than that in normal tissue imaging because the echo from blood scatterers has low correlation with interference from the cranium.

Blood flow velocity in the middle cerebral artery of a normal person is approximately $0.70 \mathrm{~m} / \mathrm{s}$ [35]. When vasospasm occurs, the velocity becomes higher than $1.0 \mathrm{~m} / \mathrm{s}$ [36]. In this study we investigated the performance of the proposed method in the setting of occurrence of vasospasm.

Figure 7 shows the beam pattern at the center frequency constructed by the conventional DAS beamformer and the proposed SDI beamformer with $K_{\text {ave }}$ of 5 , where $\mathrm{SI}_{\mathrm{C}} \mathrm{R}$ is $-40 \mathrm{~dB}$, and the directions of arrival of interferences $\theta_{i}$ are $-15,-7.5,5.0$, and 10 degrees from the front direction. The beam pattern of the proposed SDI beamformer shows nulls at all the directions of arrival of interferences. This result shows that the SDI beamformer using the proposed technique works effectively in suppressing interferences, indicating the validity of this study.

\section{Conclusion}

In this paper, we proposed a technique to improve the SDI beamformer with the Capon method for use in TCD. The proposed technique employs temporal- and axial-averaging and estimates 
the covariance matrix to improve the accuracy of the SDI beamformer in estimating blood flow velocity at the cost of axial resolution.

In this study, we used temporal and axial resolutions of $0.70 \mathrm{~ms}$ and $5.6 \mathrm{~mm}$, respectively. We investigated the performance of the SDI beamformer using the proposed technique in a simulation study under the condition that $\mathrm{SI}_{\mathrm{C}} \mathrm{R}$ was $-40 \mathrm{~dB}$ and blood flow velocity was $1.0 \mathrm{~m} / \mathrm{s}$. The conventional DAS beamformer had estimation error and standard deviation of blood flow velocity of $1.9 \mathrm{~m} / \mathrm{s}$ and $0.92 \mathrm{~m} / \mathrm{s}$, respectively. When the size for spatial averaging ranged from 3 to 6 , the proposed SDI beamformer succeeded in estimating the velocity with estimation error and standard deviation of $0.044 \mathrm{~m} / \mathrm{s}$ and $0.035 \mathrm{~m} / \mathrm{s}$, respectively, compared with $0.17 \mathrm{~m} / \mathrm{s}$ and $0.25 \mathrm{~m} / \mathrm{s}$ for the conventional SDI beamformer. The appropriate size for spatial averaging in TCD is smaller than that in normal tissue imaging, because the echo from blood scatterers has low correlation with interference from the cranium.

These results indicate that the proposed SDI beamformer has high performance in suppressing the effect of interference and may improve the accuracy of blood flow velocity estimation using TCD. We believe that the proposed SDI beamformer is suitable for high accuracy blood flow velocity estimation using TCD in postoperative management after brain surgery.

\section{Acknowledgments}

This work was partly supported by the Innovative Techno-Hub for Integrated Medical Bio-imaging Project of the Special Coordination Funds for Promoting Science and Technology, from the Ministry of Education, Culture, Sports, Science and Technology (MEXT), Japan.

\section{Conflict of Interest}

We have no conflicts of interest relationship with any companies or commercial organizations based on the definition of Japanese Society of Medical and Biological Engineering.

\section{References}

1. Frontera JA, Fernandez A, Schmidt JM, Claassen J, Wartenberg KE, Badjatia N, Connolly ES, Mayer SA: Defining vasospasm after subarachnoid hemorrhage. What is the most clinically relevant definition? Stroke. 40(6), pp. 1963-1968, 2009.

2. Dhar R, Scalfani MT, Blackburn S, Zazulia AR, Videen T, Diringer M: Relationship between angiographic vasospasm and regional hypoperfusion in aneurysmal subarachnoid hemorrhage. Stroke. 43(7), pp. 1788-1794, 2012.

3. Takagi R, Hayashi H, Kobayashi H, Kumazaki T, Isayama K, Ikeda Y, Teramoto A: Three-dimensional CT angiography of intracranial vasospasm following subarachnoid hemorrhage. Neuroradiology. 40(10), pp. 631-635, 1998.

4. Tamatani S, Sasaki O, Takeuchi S, Fujii Y, Koike T, Tanaka R: Detection of delayed cerebral vasospasm, after rupture of intracranial aneurysms, by magnetic resonance angiography. Neurosurgery. 40(4), pp. 748-754, 1997.

5. Carrera E, Schmidt JM, Oddo M, Fernandez L, Claassen J, Seder D, Lee K, Badjatia N, Connolly ES Jr., Mayer SA: Transcranial Doppler for predicting delayed cerebral ischemia after subarachnoid hemorrhage. Neurosurgery. 65(2), pp. 316-324, 2009.

6. Torp H: Clutter rejection filters in color flow imaging: a theoretical approach. IEEE Trans Ultrason Ferroelectr Freq Control.
44(2), pp. 417-424, 1997.

7. Chang L-W, Hsu K-H, Li P-C: Graphics processing unit-based high-frame-rate color Doppler ultrasound processing. IEEE Trans Ultrason Ferroelectr Freq Control. 56(9), pp. 1856-1860, 2009.

8. Takahashi H, Hasegawa H, and Kanai H: Improvement of automated identification of the heart wall in echocardiography by suppressing clutter component. Jpn J Appl Phys. 52(7S), 07HF17, 2013.

9. Okumura S, Kita A, Taki H, Sato T: Transcranial Doppler ultrasound using adaptive beamforming technique for the suppression of high-intensity interferences. Proc IEEE Ultrason Symp. pp. 1480-1483, 2013.

10. Giller CA, Giller AM: A new method for fixation of probes for transcranial Doppler ultrasound. J Neuroimaging. 7(2), pp. 103 105, 1997.

11. Bissonnette B, Stephan PN, Andras B: U.S. Patent. No. 5409005, 1995.

12. Capon J: High-resolution frequency-wavenumber spectrum analysis. Proc IEEE. 57(8), pp. 1408-1418, 1969.

13. Takao K, Kikuma N: An adaptive array utilizing an adaptive spatial averaging technique for multipath environments. IEEE Trans Antennas Propagat. 35(12), pp. 1389-1396, 1987.

14. Palmer RD, Gopalam S, Yu T-Y, Fukao S: Coherent radar imaging using the Capon's Method. Radio Sci. 33(6), pp. 1585-1598, 1998

15. Taki H, Taki K, Sakamoto T, Yamakawa M, Shiina T, Kudo M, Sato T: High range resolution ultrasonographic vascular imaging using frequency domain interferometry with the Capon method. IEEE Trans Med Imag. 31(2), pp. 417-429, 2012.

16. Synnevåg J-F, Austeng A, Holm S: Benefits of minimum-variance beamforming in medical ultrasound imaging. IEEE Trans Ultrason Ferroelectr Freq Control. 56(9), pp. 1868-1879, 2009.

17. Kimura T, Taki H, Sakamoto T, Sato T: Experimental study of high-range-resolution medical acoustic imaging for multiple target detection by frequency domain interferometry. Jpn J Appl Phys. 48(7S), 07GJ07, 2009.

18. Nilsen CC, Hafizovic I: Beamspace adaptive beamforming for ultrasound imaging. IEEE Trans Ultrason Ferroelectr Freq Control. 56(10), pp. 2187-2197, 2009.

19. Herman LH, Ostrowski AZ, Gurdjian ES: Perforating branches of the middle cerebral artery: an anatomical study. Arch Neurol. 8(1), pp. 32-34, 1963.

20. Jain KK: Some observations on the anatomy of the middle cerebral artery. Can J Surg. 7(7), pp. 134-139, 1964.

21. Teal JS, Rumbaugh CL, Bergeron RT, Segall HD: Anomalies of the middle cerebral artery: accessory artery, duplication, and early bifurcation. AJR Am J Roentgenol. 118(3), pp. 567-575, 1973.

22. Abeysekera S: Performance of pulse-pair method of Doppler estimation. IEEE Trans Aerospace Electron Syst. 34(2), pp. 520531, 1998.

23. Bossy E, Maryline T, Pascal L: Three-dimensional simulations of ultrasonic axial transmission velocity measurement on cortical bone models. J Acoust Soc Am. 115(5), pp. 2314-2324, 2004.

24. Bossy E, Padilla F, Peyrin F, Laugier P: Three-dimensional simulation of ultrasound propagation through trabecular bone structures measured by synchrotron microtomography. Phys Med Biol. 50(23), pp. 5545-5556, 2005.

25. Nagatani $Y$, Imaizumi H, Fukuda T, Matsukawa M, Watanabe $Y$, Otani T: Applicability of finite-difference time-domain method to simulation of wave propagation in cancellous bone. Jpn J Appl 
Phys. 45(9A), pp. 7186-7190, 2006.

26. Seiler RW, Grolimund P, Aaslid R, Huber P, Nornes H: Cerebral vasospasm evaluated by transcranial ultrasound correlated with clinical grade and CT-visualized subarachnoid hemorrhage. J Neurosurg. 64(4), pp. 594-600, 1986.

27. Nagatani Y, Mizuno K, Saeki T, Matsukawa M, Sakaguchi T, Hosoi H: Numerical and experimental study on the wave attenuation in bone-FDTD simulation of ultrasound propagation in cancellous bone. Ultrasonics. 48(6), pp. 607-612, 2008.

28. Nagatani Y, Mizuno K, Saeki T, Matsukawa M, Sakaguchi T, Hosoi H: Propagation of fast and slow waves in cancellous bone: Comparative study of simulation and experiment. Acoust Sci \& Tech. 30(4), pp. 257-264, 2009.

29. Nagatani Y, Mizuno K, Matsukawa M: Two-wave behavior under various conditions of transition area from cancellous bone to cortical bone. Ultrasonics. 54(5), pp. 1245-1250, 2014.

30. Moehring MA, Curry MA: U.S. Patent. No. 8162837, 2012.

31. Jensen JA: Field: A program for simulating ultrasound systems. Med Biol Eng Comput. 34(1), pp. 351-353, 1996.

32. Jensen JA, Svendsen NB: Calculation of pressure fields from arbitrarily shaped, apodized, and excited ultrasound transducers. IEEE Trans Ultrason Ferroelectr Freq Control. 39(2), pp. 262267, 1992.

33. Udesen J, Gran F, Hansen KL, Jensen JA, Thomsen C, Nielsen MB: High frame-rate blood vector velocity imaging using plane waves: simulations and preliminary experiments. IEEE Trans Ultrason Ferroelectr Freq Control. 55(8), pp. 1729-1743, 2008.

34. Ellegala DB, Leong-Poi H, Carpenter JE, Klibanov AL, Kaul S, Shaffrey ME, Sklenar J, Lindner JR: Imaging tumor angiogenesis with contrast ultrasound and microbubbles targeted to $\alpha_{\mathrm{v}} \beta_{3}$. Circulation. 108(3), pp. 336-341, 2003.

35. Krejza J, Szydlik P, Liebeskind DS, Kochanowicz J, Bronov O, Mariak Z, and Melhem ER: Age and sex variability and normal reference values for the VMCA/VICA index. AJNR Am J Neuroradiol. 26(4), pp. 730-735, 2005.

36. Badjatia N, Topcuoglu MA, Pryor JC, Rabinov JD, Ogilvy CS, Carter BS, Rordorf GA: Preliminary experience with intra-arterial nicardipine as a treatment for cerebral vasospasm. AJNR Am J Neuroradiol. 25(5), pp. 819-826, 2004.

\section{Shigeaki OKUMURA}

Shigeaki ОкuмURA received his B.E. degree from Kyoto University, Japan in 2013. He has been working about adaptive signal processing techniques for medical ultrasound imaging for his master degree in Graduate School of Informatics, Kyoto University, Japan.

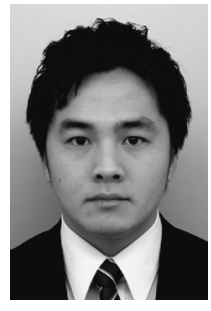

\section{Aya KITA}

Aya KITA received a M.D. degree from Kyoto University in 2000 and a Ph.D. degree from the Graduate School of Medicine at Kyoto University in 2011. She is presently a trustee at Sakai Rumi Clinic. She is qualified as Japan Neurosurgical Society Specialist Physician.

\section{Hirofumi TAKI}

Hirofumi TAKI received a M.D. degree from Kyoto University in 2000, a Ph.D. degree in informatics from Kyoto University in 2007, and is presently an assistant professor at Kyoto University. He has worked on development of the spatial resolution and calcification detection ability in Ultrasonogra-

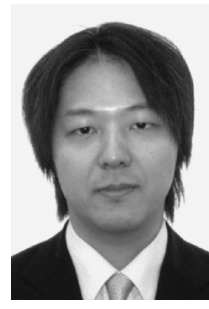
phy. IEEE, Japan Society of Ultrasonics in Medicine, Acoustic Society of Japan, The Institute of Electronics, Information and Communication Engineers, Japan Society for Medical and Biological Engineering member.

\section{Yoshiki NAGATANI}

Yoshiki NAGATANI, who is mainly working on medical engineering, acoustical simulation, and psychoacoustics, is an associate professor of Department of Electronics, Kobe City College of Technology, as well as being a visiting researcher in Université Paris-Est Créteil, France. After re-

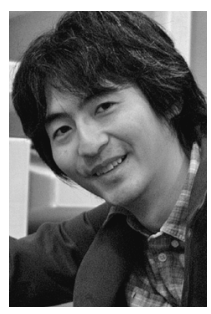
ceiving his Ph.D degree in 2006 from Doshisha University, he engaged in research projects of indoor environmental medicine and psychoacoustics at Nara Medical University focusing on human hearing mechanism, auditory brain activity, and evaluation of acoustic environment.

\section{Mami Matsukawa \\ Mami MatsuKaWA was born in Kyoto, Japan. She joined AIST (National Institute of Advanced In- dustrial Science and Technology) MITI (Ministry of International trade and Industry) in Osaka, Ja- pan. She received Doctor of Engineering degree from Doshisha University, Kyoto, Japan in 1993.

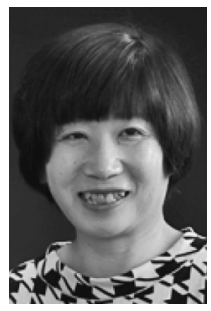 Currently, she is a professor at the Faculty of Science and Engineering, Doshisha University, Japan. Her research interests include bone quan- titative ultrasound, Brillouin scattering, pulse wave and ultrasonic elec- tronics.}

\section{Toru SATo}

Toru SATo received his B.E., M.E., and Ph.D. degrees in electrical engineering from Kyoto University, Kyoto, Japan in 1976, 1978, and 1982, respectively. He has been with Kyoto University since 1983 and is currently a Professor in the Department of Communications and Computer Engineer-

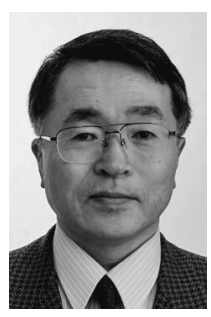
ing, Graduate School of Informatics. His major research interests include system design and signal processing aspects of UWB radars, atmospheric radars, radar remote sensing of the atmosphere, and biomedical imaging. He is a fellow of the Institute of Electronics, Information, and Communication Engineers of Japan, and a member of the Society of Geomagnetism and Earth, Planetary and Space Sciences, the Japan Society for Aeronautical and Space Sciences, the Institute of Electrical and Electronics Engineers, and American Meteorological Society. 